\title{
Impact of Preoperative Risk Factors on Morbidity after Esophagectomy: Is There Room for Improvement?
}

\author{
Styliani Mantziari • Martin Hübner • \\ Nicolas Demartines • Markus Schäfer
}

Published online: 8 July 2014

(c) Société Internationale de Chirurgie 2014

\begin{abstract}
Background Despite progress in multidisciplinary treatment of esophageal cancer, oncologic esophagectomy is still the cornerstone of therapeutic strategies. Several scoring systems are used to predict postoperative morbidity, but in most cases they identify nonmodifiable parameters. The aim of this study was to identify potentially modifiable risk factors associated with complications after oncologic esophagectomy.

Methods All consecutive patients with complete data sets undergoing oncologic esophagectomy in our department during 2001-2011 were included in this study. As potentially modifiable risk factors we assessed nutritional status depicted by body mass index (BMI) and preoperative serum albumin levels, excessive alcohol consumption, and active smoking. Postoperative complications were graded according to a validated 5-grade system. Univariate and multivariate analyses were used to identify preoperative risk factors associated with the occurrence and severity of complications.

Results Our series included 93 patients. Overall morbidity rate was $81 \%(n=75)$, with $56 \%(n=52)$ minor complications and $18 \%(n=17)$ major complications. Active smoking and excessive alcohol consumption were associated with the occurrence of severe complications,
\end{abstract}

This paper was presented in part at the 99th annual congress of the Swiss Surgical Society, June 2012, Davos, Switzerland.

S. Mantziari · M. Hübner · N. Demartines · M. Schäfer $(\bowtie)$

Department of Visceral Surgery, University Hospital CHUV, 46

Rue du Bugnon, Lausanne 1011, Switzerland

e-mail: markus.schafer@chuv.ch

S. Mantziari

e-mail: styliani.mantziari@chuv.ch whereas BMI and low preoperative albumin levels were not. The simultaneous presence of two or more of these risk factors significantly increased the risk of postoperative complications.

Conclusions A combination of malnutrition, active smoking and alcohol consumption were found to have a negative impact on postoperative morbidity rates. Therefore, preoperative smoking and alcohol cessation counseling and monitoring and improving the nutritional status are strongly recommended.

\section{Introduction}

Treatment of esophageal cancer has become a multidisciplinary task aiming to improve long-term survival. Although very early-stage and node-negative cancers can probably be treated by endoscopic approach alone, more advanced tumor stages require oncologic surgery to achieve complete (R0) resection. In the latter case, oncologic esophagectomy is often embedded in a perioperative radiochemotherapy regimen. Significant efforts have been made during recent years to reduce morbidity and mortality rates-e.g., with nutritional support, minimally invasive surgery, improved anesthesia, intensive care unit (ICU) stays [1-5]. As with other types of major gastrointestinal cancer surgery, mortality has been significantly reduced, but overall morbidity rates still approach $60 \%$ [5, 6]. Complications not only increase both hospital stay and health care costs, they strongly compromise the patient's quality of life. Recent evidence suggests that increased morbidity negatively affects long-time survival [7-11].

Early identification of patients at high risk for complications may be a promising strategy to adjust perioperative 
care and subsequently to decrease postoperative morbidity. Unfortunately, many co-morbidities that a concern for patients' general health status cannot be changed, and intraoperative risk factors are not predictable preoperatively (e.g., operative time, blood loss). On the contrary, malnutrition, increased alcohol consumption, and active smoking are common modifiable risk factors that could be corrected preoperatively in an attempt to improve the postoperative outcome $[2,6,10]$. There is good evidence that preoperative physical training, particularly inspiratory muscle training, effectively reduces postoperative complications (e.g., atelectasis formation, pneumonia, length of stay) in patients scheduled for elective cardiac surgery [12-14].

This study aimed to assess the predictive value of malnutrition, smoking, and alcohol abuse on the incidence and severity of complications after esophageal resection for cancer.

\section{Patients and methods}

From 2001 to 2011, a total of 128 patients undergoing esophageal resection for malignant disease at the Department of Visceral Surgery, University Hospital of Lausanne (CHUV) were entered into a prospectively maintained database. Patients with incomplete data sets were excluded from the current study. The Institutional Review Board approved the study protocol.

Assessment of postoperative morbidity and mortality

Postoperative morbidity included both surgical and nonsurgical complications. Every event deviating from the normal operative course was taken into account. Complications were graded according to their severity using a validated 5-point scale (grades I, II, IIIa,b, Iva,b, V) [15]. Complications requiring treatment under general anesthesia or ICU i treatment, graded IIIb to IVb, were defined as severe. Grades I, II, and IIIa were considered minor. Anastomotic leak was diagnosed based on clinical symptoms and confirmed by contrast-enhanced computed tomography. Contrast swallows were performed routinely 5 days after surgery. Postoperative surgical-site infections were actively identified during the hospital stay using the ASEPSIS definition [16]. Postoperative mortality (complication grade $\mathrm{V}$ ) was defined as patient death during the first 30 days postoperatively or during the same hospital stay as the esophageal resection (index operation).

\section{Risk factors}

The following risk factors were considered as potentially modifiable: (1) nutritional status assessed by body mass index (BMI) and preoperative serum albumin levels dating from less than 6 weeks preoperatively; (2) alcohol consumption; (3) smoking. Alcohol intake was estimated and classified according to national guidelines with the limit of excessive alcohol consumption considered to be 20 and $40 \mathrm{~g}$ of pure alcohol daily for men and women, respectively [17]. Smoking was defined as active smoking at the time of the esophageal cancer diagnosis. Serum albumin levels of $<30 \mathrm{~g} / \mathrm{L}$ and $\mathrm{BMI}<18.5 \mathrm{~kg} / \mathrm{m}^{2}$ were considered indicators of malnutrition [18-20]. Myocardial infarction as the most severe cardiovascular disease and cerebrovascular insults were not included as potential risk factors as only a few patients had such events in their medical history (five patients with myocardial infarction, three with cerebrovascular insults, two with both adverse events).

Demographic and disease-related nonmodifiable variables were included in the analysis to risk-adjust and to avoid potential confounding. They included age, American Society of Anesthesiologists (ASA) class, neoadjuvant treatment, and impaired pulmonary function tests. For the latter, forced expiratory volume in $1 \mathrm{~s}\left(\mathrm{FEV}_{1}\right)$ was chosen as the most representative test, with $<70 \%$ of the predicted value considered pathologic $[15,21,22]$.

\section{Statistical analysis}

The impact of preoperatively identified risk factors on the incidence and severity of complications after oncologic esophagectomy was assessed. Moreover, the cumulative risk in case of multiple risk factors was separately analyzed. If a patient had several complications, the highest grade was retained for further analysis.

The $\chi^{2}$ test and Fisher's exact test were used for univariate analysis to identify preoperative risk factors that could predict occurrence and severity of postoperative complications. The Mann-Whitney and Kruskal-Wallis tests were used to assess nonparametric data where appropriate. Logistic regression and multiple regression analyses were used for the multivariate analysis. Receiver operating characteristics (ROC) curve analysis was used to test the diagnostic performance of a given risk factor to discriminate patients with complications and/or severe complications from patients without complications. A value of $p<0.05$ was considered to indicate statistical significance. Data analysis was performed with MedCalc software, version 12.4.0 (MedCalc, Ostend, Belgium).

\section{Results}

In all, 93 patients fulfilling the inclusion criteria underwent esophagectomy for cancer within the defined time period. Demographics and patient-related risk factors are shown in 
Table 1 Demographics of patients with and without postoperative complications

Unless otherwise stated, results are shown as the number $(\%)$ or median (range) as appropriate

ASA American Society of Anesthesiologists, BMI body mass index, $F E V_{l}$ forced expiratory volume in $1 \mathrm{~s}$

${ }^{a}$ Proportion of ASA I/II versus. III/IV patients was compared between the two groups

b Pulmonary function was tested in only 74 of 95 patients

\begin{tabular}{lllll}
\hline Variable & $\begin{array}{l}\text { All } \\
\text { patients } \\
(n=93)\end{array}$ & $\begin{array}{l}\text { Patients } \\
\text { without complications } \\
(n=18)\end{array}$ & $\begin{array}{l}\text { Patients } \\
\text { with complications } \\
(n=75)\end{array}$ & $p$ \\
\hline Age (years) & $64(46-84)$ & $66(46-84)$ & $63(46-82)$ & 0.98 \\
Age $>65$ years & $42(45 \%)$ & $9(50 \%)$ & $33(44 \%)$ & 0.80 \\
ASA I+II & $58(62 \%)$ & $11(61 \%)$ & $47(63 \%)$ & $0.88^{\text {a }}$ \\
ASA III+IV & $35(38 \%)$ & $7(39 \%)$ & $28(37 \%)$ & \\
BMI $\left(\mathrm{kg} / \mathrm{m}^{2}\right)$ & $24(14-36)$ & $26(18-36)$ & $24(14-36)$ & 0.18 \\
BMI $<20 \mathrm{~kg} / \mathrm{m}^{2}$ & $20(22 \%)$ & $3(17 \%)$ & $17(23 \%)$ & \\
Albumin $(\mathrm{g} / \mathrm{L})$ & $38(17-49)$ & $40(24-45)$ & $37(17-49)$ & 0.46 \\
Albumin $<30 \mathrm{~g} / \mathrm{L}$ & $25(27 \%)$ & $3(17 \%)$ & $22(29 \%)$ & \\
Active smoking & $67(72 \%)$ & $10(56 \%)$ & $57(76 \%)$ & 0.10 \\
Alcohol abuse & $63(68 \%)$ & $12(67 \%)$ & $51(68 \%)$ & 0.99 \\
Abnormal pulmonary function $\left(\mathrm{FEV}_{1}\right)^{b}$ & $18(64 \%)$ & $4(22 \%)$ & $14(19 \%)$ & 0.82 \\
Preoperative chemoradiotherapy & $42(44 \%)$ & $9(50 \%)$ & $33(44 \%)$ & 0.97 \\
\hline
\end{tabular}

Table 1. Among them, $71(76.5 \%)$ patients underwent thoracoabdominal Ivor-Lewis resection with intrathoracic gastroesophageal anastomosis, mostly performed using a mechanical stapling device. Another six patients $(6.5 \%)$ had a transhiatal resection, and 16 (17\%) underwent a three-field resection (thoracoabdominal resection with cervical anastomosis) with posterior mediastinal passage of the gastric transplant. Among the 18 patients without complications, 14 (78\%) underwent Ivor-Lewis resection, two had a transhiatal resection, and two had a three-field resection. Among the 75 patients with complications, 57 (76\%) underwent Ivor-Lewis resection, four had a transhiatal resection, and 14 had a three-field resection. The overall median operative time was $380 \mathrm{~min}$ (range 195-760 min). An R0 resection was achieved in 89 patients $(94 \%)$. The median number of retrieved lymph nodes was 13 (range 0-79).

There were 35 patients with incomplete data. Most of them were excluded because of missing precise information on alcohol consumption and smoking habits.

\section{Clinical outcome}

The overall complication rate was $81 \%(n=75): 56 \%$ $(n=52)$ were minor complications, and $18 \%(n=17)$ were major complications. The mortality rate was $6 \%$ (six patients). Detailed information about the complications, by grade and category, is given in Table 2. The median length of hospital stay (LOS) was 21 days (range 10-197 days). The median stay in the ICU was 3 days (0-31 days). Whereas patients without complications had a median LOS of 15 days (range 10-26 days), patients with complications had a significantly prolonged median LOS stay of 23.5 days (range 5-197 days) ( $p=0.0005)$. Severe complications were related to an even longer median LOS of 34 days (range $10-197$ days) $(p=0.0001)$.
Table 2 Overall complications displayed by severity and by system

\begin{tabular}{ll}
\hline Complications for all patients $(n=93)$ & $\mathrm{n}(\%)$ \\
\hline None & $18(19)$ \\
Grade I & $9(10)$ \\
Grade II & $31(33)$ \\
Grade IIIA/IIIB & $12 / 5(13 / 5)$ \\
Grade IVA/IVB & $9 / 3(10 / 4)$ \\
Grade V (mortality) & $6(6)$ \\
Minor (I-IIIA) & $52(56)$ \\
Major (IIIB-IVB) & $17(18)$ \\
Pulmonary complications & $55(59)$ \\
Cardiovascular complications & $35(38)$ \\
Anastomotic leakage & $18(19)$ \\
Surgical-site infections & $8(9)$ \\
\hline
\end{tabular}

Impact of preoperative risk factors

Even though age represents a nonmodifiable parameter, it must be taken into account as a cofactor that may accentuate the risk for postoperative complications in the presence of other risk factors. In this series, increasing age was not associated with the risk to develop postoperative complications and did not correlate with the severity of complications ( $p=0.46$ ) (Table 3 ). It therefore cannot be considered a predictive factor. The median age of patients with and without complications was similar (66.0 vs. 63.5 years, $p=0.98$ ).

The BMI was not associated with increased probability for the development of postoperative complications. Even when using BMI $<18.5 \mathrm{~kg} / \mathrm{m}^{2}$ was the surrogate parameter for malnutrition, no significant increase of postoperative complication rates could be detected $(p=0.89)$. The areas under the ROC curve (AUCs) for the occurrence of overall complications and severe complications were 0.604 [95\% 
Table 3 Univariate analysis of potential parameters related to the occurrence of severe postoperative complications

\begin{tabular}{llll}
\hline Parameter & $\begin{array}{l}\text { Patients without } \\
\text { complications }\end{array}$ & $\begin{array}{l}\text { Patients with severe } \\
\text { complications }\end{array}$ & $p$ \\
\hline Age (years) & & & \\
$\quad<65$ & 8 & 13 & 0.83 \\
$>65$ & 9 & 10 & \\
BMI $\left(\mathrm{kg} / \mathrm{m}^{2}\right)$ & & & \\
$<20$ & 3 & 7 & 0.25 \\
$>20$ & 14 & 16 & \\
Albumin $(\mathrm{g} / \mathrm{l})$ & & & 0.13 \\
$<30$ & 4 & 12 & $\mathbf{0 . 0 0 2}$ \\
$>30$ & 13 & 11 & 0.21 \\
\hline Smoking & 9 & 23 & \\
Alcohol abuse & 11 & 19 & \\
\hline
\end{tabular}

confidence interval (CI) $0.497-0.704, p=0.217]$ and 0.608 (95\% CI 0.501-0.707, $p=0.107$ ) (Figs. 1, 2).

Low serum albumin levels were associated with neither the incidence nor the severity of postoperative complications. Even the cutoff of $30 \mathrm{~g} / \mathrm{dl}$ failed to reveal a significantly increased risk to develop postoperative complications or more severe complications for the low albumin group. The AUCs for the occurrence of overall complications and severe complications were 0.557 (95\% CI $0.451-0.660, \quad p=0.415)$ and $0.575 \quad(95 \%$ CI $0.468-0.677, p=0.349$ ) (Figs. 1, 2).

Increased alcohol consumption was not significantly correlated with the overall complication rate. Although univariate and multivariate analyses showed no significance for the occurrence of severe complications, the AUCs were significant, with 0.605 (95\% CI 0.498-0.705, $p=0.0310$ ).

Even though smoking was not a statistically significant factor to predict the occurrence of overall postoperative complications, it was significantly associated with the occurrence of severe complications $(p=0.002)$. This finding was confirmed by the AUC for severe complications, with 0.688 (95\% CI $0.584-0.780, p<0.0001$ ) (Fig. 2). All 23 patients with severe complications were active smokers, and all of them developed pulmonary complications. In contrast, nonsmoking patients had only mild or no complications at all. Pulmonary function was tested preoperatively in 74 patients and was considered pathologic in 18 patients $\left(\mathrm{FEV}_{1}<70 \%\right.$ of the predicted value). Among these patients, only two were nonsmokers. In this small patient group, pathologic $\mathrm{FEV}_{1}$ was not a predictive factor for postoperative complications.

The multivariate analysis regarding the occurrence of overall postoperative complications did not reveal smoking, BMI, albumin, or alcohol abuse as a significant risk factor. Smoking, however, was significantly related to severe complications (Table 4).

As shown, all of the above parameters failed to stand out as a major risk factor on their own. We therefore further assessed whether the simultaneous presence of several of these modifiable risk factors was of importance. Indeed, the risk to develop postoperative complications was significantly determined by the number of simultaneously present risk factors. The more risk factors present, the greater was the probability of developing overall and severe postoperative complications (Table 5). In particular, the presence of at least two risk factors significantly increased the chance of developing complications and severe complications. The respective AUC values were 0.630 (95\% CI 0.524-0.728, $p=0.0325)$ and 0.725 (95\% CI $0.622-0.812, p>0.0001)$ (Figs. 1, 2).

\section{Discussion}

We assessed the impact of potentially modifiable preoperative risk factors, such as malnutrition (depicted by low BMI and serum albumin), active smoking, and increased alcohol consumption on the occurrence of postoperative complications after oncologic esophagectomy. Active smoking was identified as highly predictive of the occurrence of severe postoperative complications. Increased alcohol consumption represented an increased individual risk for severe postoperative complications. Finally, the simultaneous presence of more than two of these risk factors significantly increased postoperative morbidity.

As most patients who undergo esophagectomy for cancer receive neoadjuvant treatment [23], a time frame is open to improve their individual risk profile before surgery. Risk adjustment of individual patients is therefore possible, even though major co-morbidity factors are difficult to modify. For example preexisting cardiovascular disease, pulmonary disease, and impaired kidney function are known to bes important risk factors, but usually scant improvements can be made $[2,6,24]$. Some risk factors, however, are modifiable by targeted interventions, which make their early identification and improvement an appealing strategy for improving the postoperative outcome. Malnutrition, smoking, and alcohol consumption are frequently encountered in esophageal cancer patients, and they can be easily identified by the patient's clinical history and laboratory findings. This makes them attractive as potential candidates for targeted interventions.

Among the above-mentioned potentially modifiable risk factors, active smoking stands out as the single most important one in our series. All 23 patients with severe complications were smokers. They all developed pulmonary complications, and most of them had multiple complications. These findings support the increasing evidence 
Fig. 1 Area under the receiver operating characteristic (ROC) curve for the occurrence of overall complications
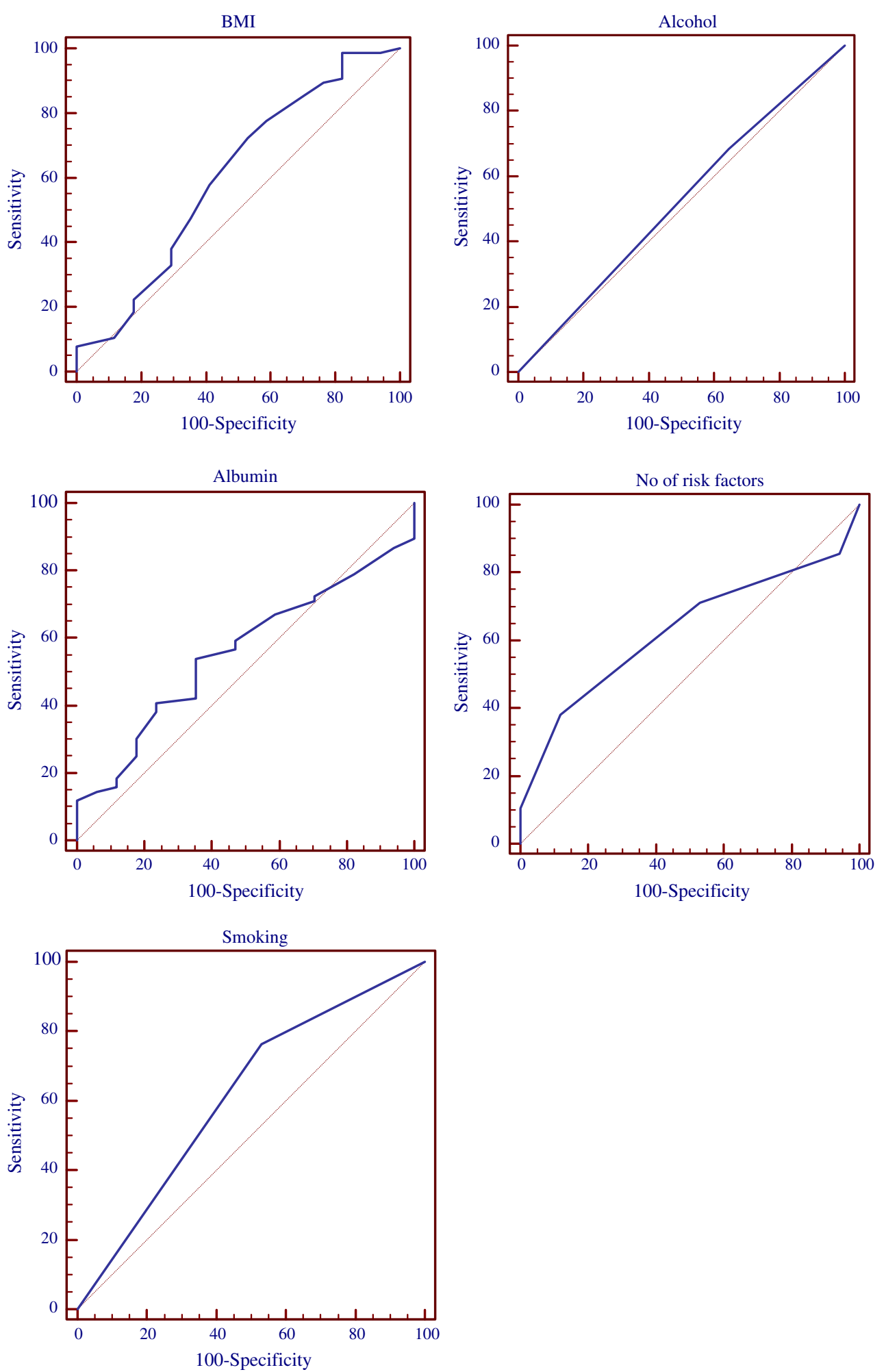

that active heavy smoking has an impact on postoperative morbidity, with the reported increase of severe complications ranging from 30 to $109 \%$ [10, 25]. Interestingly, the deleterious impact of active smoking could be alleviated by preoperative smoking cessation. It has been shown that at least 4 weeks of complete abstinence is needed to reduce respiratory complications. Also, 3 to 4 weeks of abstinence is mandatory to have a positive effect on wound healing [26]. Of note, daily smoking or a cessation of less than 3 weeks has no impact. The risk reduction is time-related, as abstinence for 8 weeks reduced the risk for respiratory complications by $47 \%$ compared to $23 \%$ for 4 weeks 
Fig. 2 Area under the ROC curve for the occurrence of severe complications
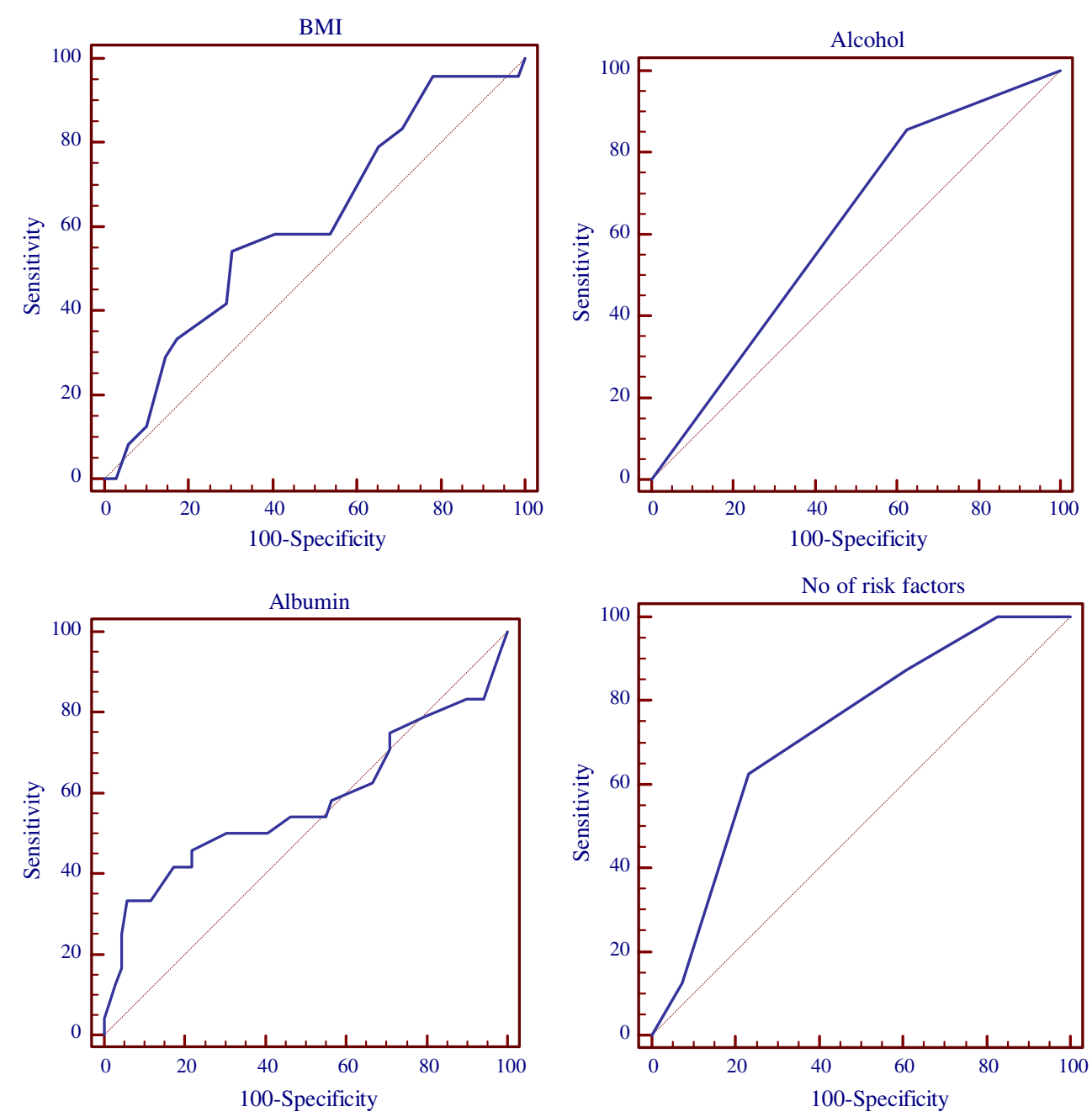

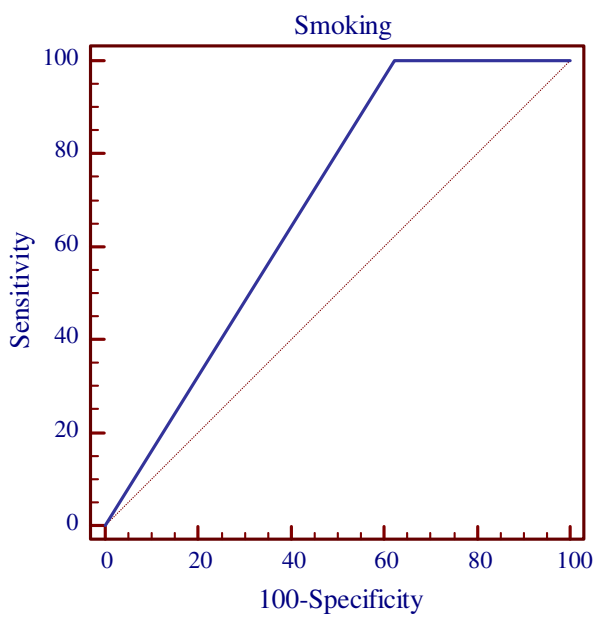

[26]. As a consequence, smoking cessation should strongly be advised to all patients with active backup from health care professionals specialized in antitobacco programs. It must also be kept in mind that long-term smoking promotes atherosclerosis, and subsequently such vascular damage probably contributes to increased postoperative morbidity rates, although its quantification may be difficult.

Upon hospital admission, an impaired nutritional status is detected in up to $50 \%$ of these patients, highlighting an important issue in daily clinical practice $[3,27]$. Because 
there is an ongoing controversy on the most appropriate methods to assess malnutrition, BMI and serum albumin levels are often used as surrogate parameters to determine patients' nutritional status [28]. In this series, low BMI did not turn out to be a significant risk factor for postoperative morbidity, as patients with BMI $<18.5 \mathrm{~kg} / \mathrm{m}^{2}$ did not develop significantly more or more-severe complications during the postoperative course. Similar findings were published in a series of 400 esophageal resections in The Netherlands where no correlation between BMI and postoperative complications was detected [29]. It must be pointed out that BMI is related, among other factors, to patients' age, physical activity, and body composition. Thus, it does not accurately represent malnutrition $[25,30]$. For future research, loss of muscle mass, known as sarcopenia, should probably be considered a better parameter for malnutrition [31]. Alternatively, the nutrition risk score (NRS) as proposed by the European Society for Parenteral and Enteral Nutrition (ESPEN) can be used [19].

Serum albumin has been used since the mid-1990's to assess patients' nutritional status and to predict short- and long-term outcomes for gastrointestinal cancer patients [18, $20,32]$. In our series, the serum albumin level was not statistically significant as a single risk factor predicting postoperative complications - as a continuous variable or

Table 4 Multivariate analysis of predictors of overall and severe complications

\begin{tabular}{llllll}
\hline \multirow{2}{*}{ Parameter } & \multicolumn{2}{l}{ Overall complications } & & \multicolumn{2}{l}{ Severe complications } \\
\cline { 2 - 3 } & $\begin{array}{l}\text { Odds } \\
\text { ratio }\end{array}$ & $95 \% \mathrm{CI}$ & & $\begin{array}{l}\text { Odds } \\
\text { ratio }\end{array}$ & $95 \% \mathrm{CI}$ \\
\hline BMI & 0.9311 & $0.8175-1.0605$ & & 0.9959 & $0.8868-1.1185$ \\
Albumin & 0.9838 & $0.9186-1.0596$ & & 0.9867 & $0.9315-1.0451$ \\
Smoking & 2.6432 & $0.7127-9.8026$ & $\mathbf{0 . 7 0 1 0}$ & $0.5970-0.7912$ \\
$\begin{array}{c}\text { Alcohol } \\
\text { abuse }\end{array}$ & 0.7022 & $0.1917-2.5726$ & & 1.1973 & $0.3237-4.4293$ \\
\hline
\end{tabular}

CI confidence interval when using the cutoff of $30 \mathrm{~g} / \mathrm{L}$ as a discriminatory level. Our results correlate well with recent findings in the literature. Changes in nutritional status are poorly represented by serum albumin levels because these levels are influenced by hydration status, active inflammation, and synthesis of acute-phase proteins [33]. Serum albumin reflects the severity of the underlying disease rather than the nutritional status. Hence, it should be used together with other criteria (e.g., the NRS) to assess preoperative malnutrition [19, 33, 34]. Postoperatively, albumin should be considered only as a negative acute-phase protein [35].

Excessive alcohol consumption has been associated with postoperative morbidity, particularly wound infections, respiratory complications, and an increased bleeding risk [36]. Complete alcohol abstinence for 4 weeks preoperatively significantly reduced the incidence of postoperative complications, although mortality rates remained unchanged [37]. Based on these considerations, alcohol was assessed in this series as a possible risk factor. With the probable bias that heavy drinkers with impaired liver function and limited compliance were a priori excluded from surgery, alcohol consumption was still found to be significant as risk factor. Especially when combined with other risk factors, its presence increased postoperative morbidity.

The risk of developing postoperative complications increased with the number of simultaneously present risk factors. In particular, the presence of two or more risk factors was highly predictive of the occurrence of severe postoperative complications as $87 \%$ of all patients with severe complications had at least two of the aforementioned risk factors.

The present study has some inherent limitations that need to be mentioned. Its retrospective character is one, although only patients with a complete data set were included. Moreover, it is a rather small series over a 10-year period, and type two errors cannot be excluded. Even if the role of the assessed risk factors may be
Table 5 Correlation between number of risk factors and occurrence of overall and severe postoperative complications
BMI, albumin, smoking, and alcohol were the possible risk factors

${ }^{\text {a }}$ The group with 0 risk factors was considered the reference for all comparisons $\left(\chi^{2}\right.$ test)

\begin{tabular}{|c|c|c|c|c|c|}
\hline $\begin{array}{l}\text { No. of risk } \\
\text { factors }\end{array}$ & $\begin{array}{l}\text { Patients without } \\
\text { complications }\end{array}$ & $\begin{array}{l}\text { Patients with minor } \\
\text { complications }\end{array}$ & $\begin{array}{l}\text { Patients with major } \\
\text { complications }\end{array}$ & Total & $p$ \\
\hline 0 & 1 & 11 & 0 & ${ }^{12}(13 \%)$ & $a$ \\
\hline 1 & 7 & 8 & 3 & $\begin{array}{l}18 \\
(19 \%)\end{array}$ & NS \\
\hline 2 & 7 & 20 & 5 & 32 & 0.05 \\
\hline 3 & 2 & 9 & 12 & $\begin{array}{l}23 \\
(25 \%)\end{array}$ & 0.05 \\
\hline 4 & 0 & 5 & 3 & $8(9 \%)$ & NS \\
\hline Total & $17(18 \%)$ & $53(57 \%)$ & $23(25 \%)$ & & \\
\hline
\end{tabular}


overestimated by a "negative" patient selection, however, the positive effects of smoking cessation and reduction of alcohol consumption have been proven to be beneficial [23, 34]. The role-specific parameters (e.g., $\mathrm{FEV}_{1}$ ) need to be investigated prospectively in larger patient series.

Treatment strategies and perioperative care have evolved during the last few years. For example, patients are now routinely screened for malnutrition, epidural analgesia is widely used, and minimally invasive surgical techniques are performed more and more often to decrease surgical trauma.

\section{Conclusions}

Targeting modifiable preoperative risk factors appears to be a valuable strategy for improving surgical outcomes. The constellation of active smoking, malnutrition, and alcohol consumption seems to have a negative impact on postoperative outcomes as they contribute to various underlying pathologies, such as decreased clearance function of bronchial epithelium, impaired liver function, microvascular disturbances and atherosclerosis, metabolic disturbances, and compromised immune function. Based on these results, a prospective trial including a large patient number is mandatory to test further the efficacy of preoperative counseling for prompt cessation of alcohol consumption and smoking as well as treatment of malnutrition in esophageal cancer patients.

Conflict of interest None.

\section{References}

1. Mariette C (2008) [Practical considerations in prescribing immunonutrition]. J Chir (Paris) 145(Suppl 4):9S 10-9S 14

2. Tekkis PP, McCulloch P, Poloniecki JD et al (2004) Riskadjusted prediction of operative mortality in oesophagogastric surgery with O-POSSUM. Br J Surg 91:288-295

3. Cerantola Y, Hübner M, Grass F et al (2011) Immunonutrition in gastrointestinal surgery. Br J Surg 98:37-48

4. Agnelli G, Eriksson BI, Cohen AT et al (2009) Safety assessment of new antithrombotic agents: lessons from the EXTEND study on ximelagatran. Thromb Res 123:488-497

5. McCulloch P, Ward J, Tekkis PP et al (2003) Mortality and morbidity in gastro-oesophageal cancer surgery: initial results of ASCOT multicentre prospective cohort study. BMJ 327: 1192-1197

6. Lagarde SM, Reitsma JB, Maris AK et al (2008) Preoperative prediction of the occurrence and severity of complications after esophagectomy for cancer with use of a nomogram. Ann Thorac Surg 85:1938-1945

7. Derogar M, Orsini N, Sadr-Azodi O et al (2012) Influence of major postoperative complications on health-related quality of life among long-term survivors of esophageal cancer surgery. J Clin Oncol 30:1615-1619
8. Dimick JB, Weeks WB, Karia RJ et al (2006) Who pays for poor surgical quality? Building a business case for quality improvement. J Am Coll Surg 202:933-937

9. Khan NA, Quan H, Bugar JM et al (2006) Association of postoperative complications with hospital costs and length of stay in a tertiary care center. J Gen Intern Med 21:177-180

10. Turan A, Mascha EJ, Roberman D et al (2011) Smoking and perioperative outcomes. Anesthesiology 114:837-846

11. Vonlanthen R, Slankamenac K, Breitenstein S et al (2011) The impact of complications on costs of major surgical procedures: a cost analysis of 1200 patients. Ann Surg 254:907-913

12. Hoogeboom TJ, Dronkers JJ, Hulzebos EH et al (2014) Merits of exercise therapy before and after major surgery. Curr Opin Anaesthesiol 27:161-166

13. Hulzebos EH, Elders PJ, Favié NJ et al (2006) Preoperative intensive inspiratory muscle training to prevent postoperative pulmonary complications in high-risk patients undergoing CABG surgery: a randomized clinical trial. JAMA 296:1851-1857

14. Hulzebos EH, Smit Y, Helders PP, et al (2012) Preoperative physical therapy for elective cardiac surgery patients. Cochrane Database Syst Rev 11:CD010118

15. Dindo D, Demartines N, Clavien PA (2004) Classification of surgical complications: a new proposal with evaluation in a cohort of 6336 patients and results of a survey. Ann Surg 240:205-213

16. Wilson AP, Treasure T, Sturridge MF et al (1986) A scoring method (ASEPSIS) for postoperative wound infections for use in clinical trials of antibiotic prophylaxis. Lancet 1:311-313

17. Swiss Federal Commission for Alcohol Problems. Available at: http://www.addictionsuisse.ch/faits-et-chiffres/alcool/consomma tion/consommation-a-risque

18. Chwals WJ, Blackburn GL (1986) Perioperative nutritional support in the cancer patient. Surg Clin N Am 66:1137-1165

19. Kondrup J, Allison SP, Elia M et al (2003) ESPEN guidelines for nutrition screening 2002. Clin Nutr 22:415-421

20. Pacelli F, Bossola M, Rosa F et al (2008) Is malnutrition still a risk factor of postoperative complications in gastric cancer surgery? Clin Nutr 27:398-407

21. Grotenhuis BA, Winjhoven BP, Grüne F et al (2010) Preoperative risk assessment and prevention of complications in patients with esophageal cancer. J Surg Oncol 101:270-278

22. Kuwano H, Sumiyoshi K, Sonoda K et al (1998) Relationship between preoperative assessment of organ function and postoperative morbidity in patients with oesophageal cancer. Eur J Surg 164:581-586

23. Pennathur A, Gibson MK, Jobe BA et al (2013) Oesophageal carcinoma. Lancet 381:400-412

24. Haga Y, Wada Y, Takeuchi H et al (2004) Estimation of physiologic ability and surgical stress (E-PASS) for a surgical audit in elective digestive surgery. Surgery 135:586-594

25. Zingg U, Smithers BM, Gotley DC et al (2011) Factors associated with postoperative pulmonary morbidity after esophagectomy for cancer. Ann Surg Oncol 18:1460-1468

26. Wong J, Lam DP, Abrishami A et al (2012) Short-term preoperative smoking cessation and postoperative complications: a systematic review and meta-analysis. Can J Anaesth 59:268-279

27. Kyle UG, Pirlich M, Schuetz T et al (2003) Prevalence of malnutrition in 1760 patients at hospital admission: a controlled population study of body composition. Clin Nutr 22:473-481

28. Schiesser M, Kirchhoff P, Müller MK et al (2009) The correlation of nutrition risk index, nutrition risk score, and bioimpedance analysis with postoperative complications in patients undergoing gastrointestinal surgery. Surgery 145:519-526

29. Han-Geurts IJ, Hop WC, Tran TC et al (2006) Nutritional status as a risk factor in esophageal surgery. Dig Surg 23:159-163 
30. Nass R, Johannsson G, Christiansen JS et al (2009) The aging population-is there a role for endocrine interventions? Growth Horm IGF Res 19:89-100

31. Sheetz KH, Zhao L, Holcombe SA et al (2013) Decreased core muscle size is associated with worse patient survival following esophagectomy for cancer. Dis Esophagus 26:716-722

32. Lien YC, Hsieh CC, Wu YC et al (2004) Preoperative serum albumin level is a prognostic indicator for adenocarcinoma of the gastric cardia. J Gastrointest Surg 8:1041-1048

33. Gupta D, Lis CG (2010) Pretreatment serum albumin as a predictor of cancer survival: a systematic review of the epidemiological literature. Nutr J 9:69
34. Kondrup J, Rasmussen HH, Hamberg O et al (2003) Nutritional risk screening (NRS 2002): a new method based on an analysis of controlled clinical trials. Clin Nutr 22:321-336

35. Kudsk KA, Tolley EA, DeWitt RC et al (2003) Preoperative albumin and surgical site identify surgical risk for major postoperative complications. JPEN J Parenter Enteral Nutr 27:1-9

36. Oppedal K, Møller AM, Pedersen B, et al (2012) Preoperative alcohol cessation prior to elective surgery. Cochrane Database Syst Rev 7:CD008343

37. Tonnesen H, Rosenberg J, Nielsen HJ et al (1999) Effect of preoperative abstinence on poor postoperative outcome in alcohol misusers: randomised controlled trial. BMJ 318:1311-1316 